PHYSICAL REVIEW A 82, 032120 (2010)

\title{
Sagnac interferometry as a probe to the commutation relation of a macroscopic quantum mirror
}

\author{
Ran Yang \\ Physics Department, Huazhong University of Science and Technology, Wuhan 430074, China \\ Xuefei Gong \\ Institute of High Energy Physics, Theoretical Physics Center for Science Facilities, Chinese Academy of Sciences, Beijing 100049, China \\ Shouyong Pei \\ Physics Department, Beijing Normal University, Beijing 100875, China \\ Ziren Luo* \\ Institute of Mechanics, Chinese Academy of Sciences, 15, Beisihuanxi road, Beijing 100190, China \\ Y. K. Lau \\ Institute of Applied Mathematics, Academy of Mathematics and System Science, Chinese Academy of Sciences, \\ 55, Zhongguancun Donglu, Beijing 100190, China \\ (Received 15 July 2010; published 30 September 2010)
}

\begin{abstract}
Single photon Sagnac interferometry as a probe to macroscopic quantum mechanics is considered at the theoretical level. For a freely moving macroscopic quantum mirror susceptible to radiation pressure force inside a Sagnac interferometer, a careful analysis of the input-output relation reveals that the particle spectrum readout at the bright and dark ports encode information concerning the noncommutativity of position and momentum of the macroscopic mirror. A feasible experimental scheme to probe the commutation relation of a macroscopic quantum mirror is outlined to explore the possible frontier between classical and quantum regimes. In the Appendix, the case of Michelson interferometry as a feasible probe is also sketched.
\end{abstract}

DOI: 10.1103/PhysRevA.82.032120

PACS number(s): 03.65.Ta, 03.65.Yz, 42.50.Ct, 42.50.Xa

\section{INTRODUCTION}

In our quest for a better understanding of quantum mechanics at the macroscopic level [1,2] and certain foundational issues involving the possible resolution of the measurement problem in terms of quantum gravity [3], optical interferometric sensing provides a viable experimental means to probe the linear superposition and quantum entanglement of macroscopic quantum mirrors [4-7]. The theme of the present work is to show that Sagnac interferometry may also be exploited to probe the commutation relation between two conjugate variables (position and momentum) of a quantum macroscopic mirror and explore the possible frontier between classical and quantum physics. The experimental barriers are no more difficult (nor easier) than the theoretical schemes suggested earlier to understand the linear superposition and nonlocal aspects of macroscopic quantum mechanics $[4,8,9]$. For the design of other experimental schemes to probe the commutation relation of more conventional (microscopic) quantum systems, see [10,11].

Our work was originally motivated by the attempt to understand Sagnac interferometer as a possible quantum nondemolition (QND) speed meter relevant to the development of advanced gravitational wave detector $[12,13]$. Then a careful analysis of the input-output relation of the interferometer at the quantum level revealed that in the simple case of one movable mirror subject to single photon radiation pressure within the ring shaped interferometer, the particle spectrum

\footnotetext{
*Corresponding author: rodgy@ 163.com
}

at the bright or dark output port registered by a photodiode actually encode information concerning the commutation relation of the position and momentum of the macroscopic quantum mirror. Later on we further realized that, though Saganc interferometry offers very different physical insights from those of Michelson interferometry at the quantum level, modulo certain technical differences, a similar conclusion may also be drawn for a Michelson interferometer and is already implicit in [4]. This will be presented in the Appendix. As Sagnac and Michelson are the two mainstream optical topologies under consideration as blueprints for the third generation of advanced gravitational wave detector [14,15], interferometric sensing method to probe the commutation relation of a quantum macroscopic mirror may be considered in the prototype testing stage of the advanced gravitational wave detector development for both optical topologies.

The main content of this short article is to sketch the derivation of the input-output relation for single photon Sagnac interferometry in the optical setup to be described in what follows. Then we go on to explain how the particle spectrum readout at the output ports may be used as a probe to the commutation relation of a macroscopic quantum mirror. In the calculations to be presented in what follows, we shall consider a very idealised situation and then come back to the possible experimental details toward the end. Throughout we shall adopt units in which $\hbar=c=1$.

\section{SAGNAC INTERFEROMETRY}

A schematic diagram of a Sagnac interferometer to be considered is shown in Fig. 1 [12,16,17]. A high finesse 


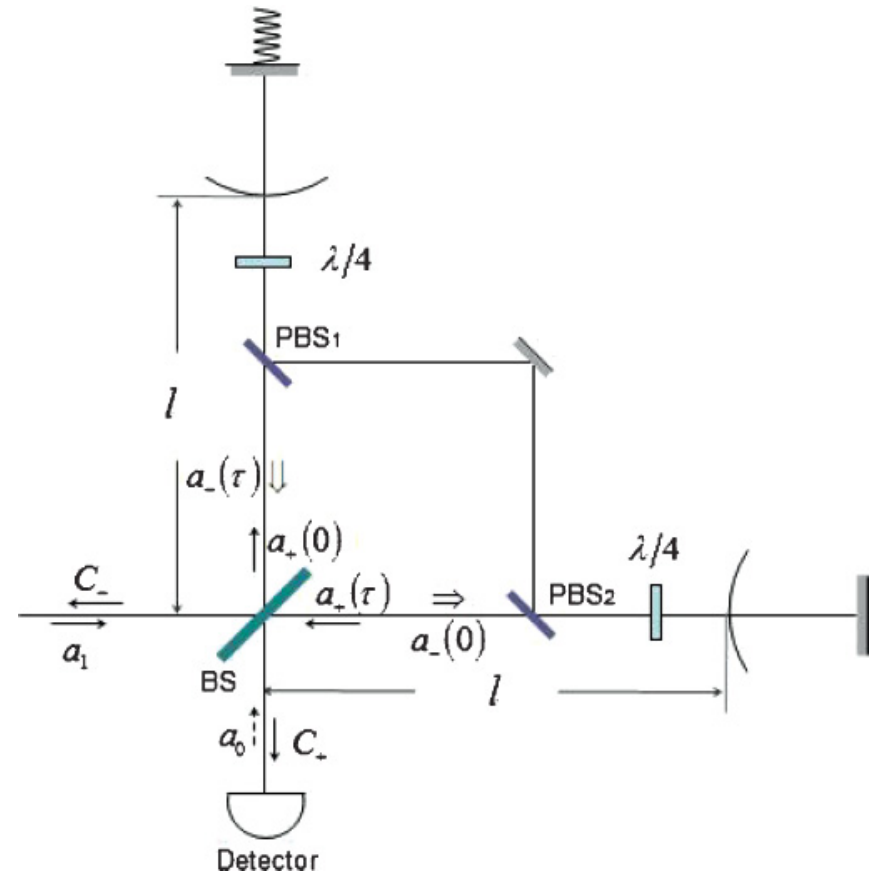

FIG. 1. (Color online) Sagnac interferometry with two cavity on the north and east arms separately, and mirror at the end of north cavity is a movable mirror.

(Fabry-Perot) optical cavity with one movable and one rigidly fixed mirror and of length $d$ are inserted in the north arm of the ring shaped interferometer, within which optomechanical coupling between the movable mirror and a single photon takes place. The end mirror in the north arm is of mass $m$, movable and subject to the single photon radiation pressure force while the mirrors in the east arm are rigidly attached. The frequency of the photon is $\omega$ and the center-of-mass motion of the mirror is modelled as a harmonic oscillator with natural oscillating frequency $\omega_{m}$. Like in the detection of gravitational waves, we assume $\omega_{m} \ll \omega$.

Upon passing through the beam splitter, a linearly polarized single photon will propagate inside the interferometer in clockwise or counterclockwise direction. For both paths taken, a single photon will enter the optical cavity in the north arm and couple to the movable mirror. However, due to the optical setup, there is a certain time delay between the clockwise and counterclockwise propagating photon in the switching on of the optomechanical coupling with the movable mirror. As we shall see, the delay time may be fine tuned by adjusting the length parameter $l$ of the interferometer (see Fig. 1) in our proposed experiment.

Let $a_{1}, a_{0}$ be the annihilation operators of single photon input field and vacuum input field (see Fig. 1) and $a_{+}, a_{-}$ the annihilation operators of the field modes propagating in the clockwise and anticlockwise direction. With a balanced (50:50) beamsplitter, we then have

$$
\left(\begin{array}{l}
a_{+} \\
a_{-}
\end{array}\right)=\frac{1}{\sqrt{2}}\left(\begin{array}{cc}
1 & 1 \\
1 & -1
\end{array}\right)\left(\begin{array}{l}
a_{1} \\
a_{0}
\end{array}\right) .
$$

Within the cavity in the north arm, a photon will exert a mechanical force on the movable mirror and the center of mass of the mirror undergoes forced oscillation. Subject to the approximations that the displacement of the mirror is sufficiently small in comparison with the length of the cavity and the cavity mode as well as the storage time remain unchanged up to second order in perturbation, the interaction Hamiltonians describing the optomechanical coupling of the clockwise and counter-clockwise propagating photon within the cavity in the north arm are given by [8]

$$
V_{ \pm}=-g a_{ \pm}^{\dagger} a_{ \pm}\left(b^{\dagger}+b\right)
$$

where $g=(\omega / d) \sqrt{\left(1 / 2 m \omega_{m}\right)}$ is the coupling constant, $b, b^{\dagger}$ are annihilation and creation operators for the phonon field mode associated with the motion of the center of mass of the mirrors.

Due to the optical topology unique to Sagnac interferometry, a clockwise propagating photon will be within the cavity during the time interval $l<t<l+\tau_{s}$, with $\tau_{s}$ the photon storage time in the cavity and the moment at which the photon entering the interferometer is chosen as the time origin. For the counterclockwise propagating photon, it will be within the cavity at the time interval $3 l+\tau_{s}<t<3 l+2 \tau_{s}$. This means that, as a function of time, the interaction term $V_{+}$will acquire nonzero support only when $l<t<l+\tau_{s}$, while $V_{-}$will have nonzero support at $3 l+\tau_{s}<t<3 l+2 \tau_{s}$. The time delay of optomechanical coupling between two possible paths traversed by a single photon distinguishes Sagnac interferometry from other optical topologies, like, for instance, the familiar Michelson interferometry.

In terms of the step function defined as

$$
\theta\left(t_{1}, t_{2}\right)= \begin{cases}1, & t_{1}<t<t_{2}, \\ 0, & \text { otherwise, }\end{cases}
$$

the finite support in time of $V_{ \pm}$at two different time intervals may be described by writing the total Hamiltonian as

$$
H=H_{0}+V_{+} \theta\left(l, l+\tau_{s}\right)+V_{-} \theta\left(3 l+\tau_{s}, 3 l+2 \tau_{s}\right),
$$

where the free Hamiltonian $H_{0}$ is given by

$$
H_{0}=\omega a_{+}^{\dagger} a_{+}+\omega a_{-}^{\dagger} a_{-}+\omega_{m} b^{\dagger} b .
$$

The insertion of step function in (2) may be regarded as an idealisation that the build up of the standing wave structure of the photon within the cavity is instantaneous.

In the next step, we shall work out the Heisenberg equations of motion for $a_{ \pm}$and $b$. Subject to the time evolution governed by the Hamiltonian in (2), we find

$$
\begin{gathered}
\dot{a}_{+}=-i \omega a_{+}+i g a_{+}\left(b^{\dagger}+b\right) \theta\left(l, l+\tau_{s}\right), \\
\dot{a}_{-}=-i \omega a_{-}+i g a_{-}\left(b^{\dagger}+b\right) \theta\left(3 l+\tau_{s}, 3 l+2 \tau_{s}\right), \\
\dot{b}=-i \omega_{m} b+i g a_{+}^{\dagger} a_{+} \theta\left(l, l+\tau_{s}\right) \\
+i g a_{-}^{\dagger} a_{-} \theta\left(3 l+\tau_{s}, 3 l+2 \tau_{s}\right) .
\end{gathered}
$$

It may also be checked from (1), (2), and (3) that

$$
\left[a_{+}^{\dagger} a_{+}, H\right]=\left[a_{-}^{\dagger} a_{-}, H\right]=0
$$

and therefore the photon number operators $a_{+}^{\dagger} a_{+}$and $a_{-}^{\dagger} a_{-}$ are conserved and equivalent to QND observables though the character of back-action-free measurement is not obvious. 
This implies that, as a harmonic oscillator, the center of mass of the mirror is under a constant radiation pressure force $g a_{+}^{\dagger} a_{+} \sqrt{2 m \omega_{m}}$ or $g a_{-}^{\dagger} a_{-} \sqrt{2 m \omega_{m}}$ at different time intervals. The conservation of $a_{ \pm}^{\dagger} a_{ \pm}$also enables us to decouple the equation of motion for $b$ from those for $a_{ \pm}$, and (4c) may be integrated directly to obtain

$$
\begin{aligned}
b(t)= & b(0) e^{-i \omega_{m} t}+e^{-i \omega_{m} t} \int_{0}^{t} e^{i \omega_{m} t^{\prime}} i g a_{+}^{\dagger} a_{+} \theta\left(l, l+\tau_{s}\right) d t^{\prime} \\
& +e^{-i \omega_{m} t} \int_{0}^{t} e^{i \omega_{m} t^{\prime}} i g a_{-}^{\dagger} a_{-} \theta\left(3 l+\tau_{s}, 3 l+2 \tau_{s}\right) d t^{\prime} .
\end{aligned}
$$

From (4a), (4b), and (5), we also have

$$
\begin{gathered}
a_{+}(t)=a_{+}(0) e^{-i \omega t+\int_{0}^{t} i g\left[b^{\dagger}\left(t^{\prime}\right)+b\left(t^{\prime}\right)\right] \theta\left(l, l+\tau_{s}\right) d t^{\prime}}, \\
a_{-}(t)=a_{-}(0) e^{-i \omega t+\int_{0}^{t} i g\left[b^{\dagger}\left(t^{\prime}\right)+b\left(t^{\prime}\right)\right] \theta\left(3 l+\tau_{s}, 3 l+2 \tau_{s}\right) d t^{\prime}} .
\end{gathered}
$$

Given $4 l+2 \tau_{s}$ is the time taken for a single photon to complete a round trip in the interferometer and we are interested in the readout at the output ports at a late time $\tau>4 l+2 \tau_{s}$, integrating (5) and (6) from $t=0$ to $\tau>4 l+2 \tau_{s}$, we have

$$
\begin{aligned}
a_{+}(\tau)= & a_{+}(0) e^{-i \omega \tau} e^{\kappa\left[b^{\dagger}(0) e^{i \omega_{m} l}\left(e^{i \omega_{m} \tau_{s}}-1\right)-b(0) e^{-i \omega_{m} l}\left(e^{-i \omega_{m} \tau_{s}}-1\right)\right]} \\
& \times e^{i 2 \kappa^{2} a_{+}^{\dagger} a_{+}\left(\omega_{m} \tau_{s}-\sin \omega_{m} \tau_{s}\right)} \\
a_{-}(\tau)= & a_{-}(0) e^{-i \omega \tau} \\
& \times e^{\kappa\left[b^{\dagger}(0) e^{i \omega_{m}\left(3 l+\tau_{s}\right)}\left(e^{i \omega_{m} \tau_{s}}-1\right)-b(0) e^{i \omega_{m}\left(3 l+\tau_{s}\right)}\left(e^{-i \omega_{m} \tau_{s}}-1\right)\right]} \\
& \times e^{\kappa^{2} a_{+}^{\dagger} a_{+}\left[\left(1-e^{-i \omega_{m} \tau_{s}}\right)^{2} e^{-i \omega_{m} 2 l}-\left(1-e^{i \omega_{m} \tau_{s}}\right)^{2} e^{i \omega_{m} 2 l}\right]} \\
& \times e^{i 2 \kappa^{2} a_{-}^{\dagger} a_{-}\left(\omega_{m} \tau_{s}-\sin \omega_{m} \tau_{s}\right)}
\end{aligned}
$$

$$
\begin{aligned}
b(\tau)= & b(0) e^{-i \omega_{m} \tau}+\kappa a_{+}^{\dagger} a_{+}\left(1-e^{-i \omega_{m} \tau_{s}}\right) e^{-i \omega_{m}\left(\tau-l-\tau_{s}\right)} \\
& +\kappa a_{-}^{\dagger} a_{-}\left(1-e^{-i \omega_{m} \tau_{s}}\right) e^{-i \omega_{m}\left(\tau-3 l-2 \tau_{s}\right)}
\end{aligned}
$$

where $\kappa=g / \omega_{m}$.

Denote by $c_{+}, c_{-}$the annihilation operators of the field modes emerging at the bright $(+)$ and dark (-) ports. Again with a balanced beamsplitter, we have

$$
\left(\begin{array}{l}
c_{+}(\tau) \\
c_{-}(\tau)
\end{array}\right)=\frac{1}{\sqrt{2}}\left(\begin{array}{cc}
1 & 1 \\
1 & -1
\end{array}\right)\left(\begin{array}{l}
a_{+}(\tau) \\
a_{-}(\tau)
\end{array}\right) .
$$

Define the number operator

$$
N_{ \pm}=c_{ \pm}^{\dagger} c_{ \pm}
$$

describing the particle spectrum registered by the photodiodes at the corresponding output ports.

From (7) and (8), it may then be inferred that

$$
\begin{aligned}
N_{ \pm}(\tau)=\frac{1}{4}\{ & a_{+}^{\dagger}(0) a_{+}(0)+a_{-}^{\dagger}(0) a_{-}(0) \\
\pm & e^{\kappa\left[b(0) e^{-i \omega_{m} l}\left(e^{-i \omega_{m} \tau_{s}}-1\right)-b^{\dagger}(0) e^{i \omega_{m} l}\left(e^{i \omega_{m} \tau_{s}}-1\right)\right]} \\
& \times e^{\left.\kappa\left[b^{\dagger}(0) e^{i \omega_{m}\left(3 l+\tau_{s}\right.}\right)\left(e^{i \omega_{m} \tau_{s}}-1\right)-b(0) e^{i \omega_{m}\left(3 l+\tau_{s}\right)}\left(e^{-i \omega_{m} \tau_{s}}-1\right)\right]} \\
& \times e^{-i 2 \kappa^{2} a_{+}^{\dagger} a_{+}\left(\omega_{m} \tau_{s}-\sin \omega_{m} \tau_{s}\right)} a_{+}^{\dagger}(0) a_{-}(0) \\
& \times e^{\kappa^{2} a_{+}^{\dagger} a_{+}\left[\left(1-e^{-i \omega_{m} \tau_{s}}\right)^{2} e^{-i \omega_{m} 2 l}-\left(1-e^{i \omega_{m} \tau_{s}}\right)^{2} e^{i \omega_{m} 2 l}\right]} \\
& \times e^{i 2 \kappa^{2} a_{-}^{\dagger} a_{-}\left(\omega_{m} \tau_{s}-\sin \omega_{m} \tau_{s}\right)} \\
\pm & \text { Hermitian conjugate } .
\end{aligned}
$$

To further compute $N_{ \pm}$, we note that, with the help of the Baker-Campell-Hausdorff formula, in (10) we may further elaborate the term

$$
\begin{aligned}
& e^{\kappa\left[b(0) e^{-i \omega_{m} l}\left(e^{-i \omega_{m} \tau_{s}}-1\right)-b^{\dagger}(0) e^{i \omega_{m} l}\left(e^{i \omega_{m} \tau_{s}}-1\right)\right]} e^{\kappa\left[b^{\dagger}(0) e^{i \omega_{m}\left(3 l+\tau_{s}\right)}\left(e^{i \omega_{m} \tau_{s}}-1\right)-b(0) e^{i \omega_{m}\left(3 l+\tau_{s}\right)}\left(e^{-i \omega_{m} \tau_{s}}-1\right)\right]} \\
& =e^{\kappa b^{\dagger}(0)\left(e^{i \omega_{m} \tau_{s}}-1\right)\left[e^{i \omega_{m}\left(3 l+\tau_{s}\right)}-e^{i \omega_{m} l}\right]} e^{-\kappa b(0)\left(e^{-i \omega_{m} \tau_{s}}-1\right)\left[e^{-i \omega_{m}\left(3 l+\tau_{s}\right)}-e^{-i \omega_{m} l}\right]} e^{2 \kappa^{2}\left(1-\cos \omega_{m} \tau_{s}\right) \sin \omega_{m}\left(2 l+\tau_{s}\right)\left[x_{0}, p_{0}\right]} e^{2 i \kappa^{2}\left(1-\cos \omega_{m} \tau_{s}\right)\left[1-\cos \omega_{m}\left(2 l+\tau_{s}\right)\right]\left[x_{0}, p_{0}\right]} \\
& =e^{-2 \kappa^{2}\left(1-\cos \omega_{m} \tau_{s}\right)\left[1-\cos \omega_{m}\left(2 l+\tau_{s}\right)-i \sin \omega_{m}\left(2 l+\tau_{s}\right)\right]} e^{\kappa b^{\dagger}(0) e^{i \omega_{m} l}\left(e^{i \omega_{m} \tau_{s}}-1\right)\left[e^{i \omega_{m}\left(2 l+\tau_{s}\right)}-1\right]} e^{-\kappa b(0)\left(e^{-i \omega_{m} \tau_{s}}-1\right)\left[e^{-i \omega_{m}\left(3 l+\tau_{s}\right)}-e^{-i \omega_{m} l}\right]},
\end{aligned}
$$

where

$x_{0}=\sqrt{\frac{1}{2 m \omega_{m}}}\left[b^{\dagger}(0)+b(0)\right], \quad p_{0}=i \sqrt{\frac{m \omega_{m}}{2}}\left[b^{\dagger}(0)-b(0)\right]$.

The scalar term appearing in the final expression in (11) follows from the commutation relation of the macroscopic mirror $\left[x_{0}, p_{0}\right]=i$. As only $x_{0}$ and $p_{0}$ are involved, it is the commutation relation of a free macroscopic mirror that eventually enters into the particle spectrum at the readout. Further inserting (11) back into $N_{ \pm}$in (10), we find

$$
\begin{aligned}
N_{ \pm}(\tau)=\frac{1}{4}\{ & a_{+}^{\dagger}(0) a_{+}(0)+a_{-}^{\dagger}(0) a_{-}(0) \\
\pm & e^{-2 \kappa^{2}\left(1-\cos \omega_{m} \tau_{s}\right)\left[1-\cos \omega_{m}\left(2 l+\tau_{s}\right)-i \sin \omega_{m}\left(2 l+\tau_{s}\right)\right]} \\
& \times\left[e^{\kappa b^{\dagger}(0) e^{i \omega_{m} l}\left(e^{i \omega_{m} \tau_{s}}-1\right)\left[e^{i \omega_{m}\left(2 l+\tau_{s}\right)}-1\right]}\right. \\
& \times e^{-\kappa b(0) e^{-i \omega_{m} l}\left(e^{-i \omega_{m} \tau_{s}}-1\right)\left[e^{-i \omega_{m}\left(2 l+\tau_{s}\right)}-1\right]} \\
& \times e^{-i 2 \kappa^{2} a_{+}^{\dagger} a_{+}\left(\omega_{m} \tau_{s}-\sin \omega_{m} \tau_{s}\right)} a_{+}^{\dagger}(0) a_{-}(0) \\
& \times e^{i 2 \kappa^{2} a_{-}^{\dagger} a_{-}\left(\omega_{m} \tau_{s}-\sin \omega_{m} \tau_{s}\right)}
\end{aligned}
$$

$$
\begin{aligned}
& \left.\times e^{\kappa^{2} a_{+}^{\dagger} a_{+}\left[-\left(1-e^{i \omega_{m} \tau_{s}}\right)^{2} e^{i \omega_{m} 2 l}+\left(1-e^{-i \omega_{m} \tau_{s}}\right)^{2} e^{-i \omega_{m} 2 l}\right]}\right] \\
\pm & \text { Hermitian conjugate }\} .
\end{aligned}
$$

Consider now the mirror in its ground state $|0\rangle_{m}$. The initial state of the coupled photon-mirror system is then $\frac{1}{\sqrt{2}}\left(|1\rangle_{+}|0\rangle_{-}+|0\rangle_{+}|1\rangle_{-}\right)|0\rangle_{m}$. When taking the expectation value of $N_{ \pm}$with respect to the coupled photon-mirror state, we find

$$
\left\langle a_{ \pm}^{\dagger}(0) a_{ \pm}(0)\right\rangle=1 / 2
$$

and the rest of the operator terms in (12) yield the unit expectation value. As a result, we have, from (12),

$$
\begin{aligned}
\left\langle N_{ \pm}(\tau)\right\rangle= & \frac{1}{2} \pm \frac{1}{2} e^{-2 \kappa^{2}\left(1-\cos \omega_{m} \tau_{s}\right)\left(1-\cos \omega_{m}\left(2 l+\tau_{s}\right)\right)} \\
& \times \cos \left[2 \kappa^{2}\left(1-\cos \omega_{m} \tau_{s}\right) \sin \omega_{m}\left(2 l+\tau_{s}\right)\right]
\end{aligned}
$$

and the particle spectrum at both outport ports are entirely determined by the real part of the scalar term generated by the 


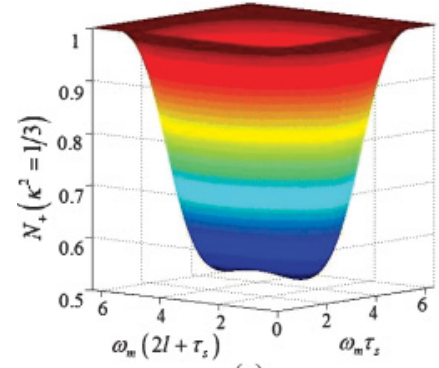

(a)

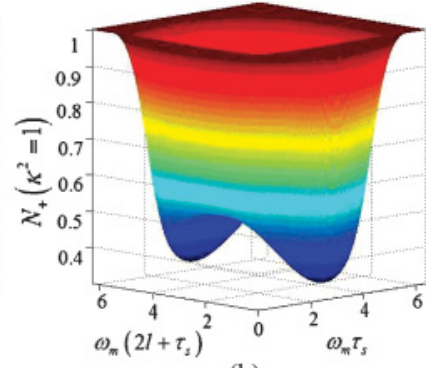

(b)
FIG. 2. (Color online) Expectation value of $N_{+}$, as a function of $\kappa, \omega_{m} \tau_{s}, \omega_{m}\left(2 l+\tau_{s}\right)$. (a) $\kappa^{2}=1 / 3$; (b) $\kappa^{2}=1$.

commutation relation of a free macroscopic mirror displayed in (11). This means that a detection of photon at the outport ports in accordance with the particle spectrum described in (13) is a direct verification of the commutation relation between the two conjugate variables $x$ and $p$ of a macroscopic mirror. This constitutes another feasible test of the validity of macroscopic quantum mechanics. Moreover, by possibly varying the size or other physical parameters of the macroscopic mirror, it is conceivable that we might also further explore the possible existence of a frontier separating the classical and quantum regime. In a more general case when the mirror is not in its ground state, one would expect to uphold the conclusion that the particle spectrum encodes information concerning the commutation relation of a macroscopic mirror, albeit in a more complicated looking expectation value of the number operator. This expectation is indeed substantiated in the case when the mirror is in a semi-classical coherent state and this will be further elaborated elsewhere [18].

As may be seen from (13), the particle spectrum at the outport ports are dependent on the experimental accessible parameters $\kappa, l$, and $\tau_{s}$. For instance, set $\cos \omega_{m} \tau_{s}=$ $\cos \omega_{m}\left(2 l+\tau_{s}\right)=-1$ and $\kappa^{2}=1 / 3$ or $\kappa^{2}=1$, respectively, we will have a $50 \%$ chance to detect a single photon at both the bright and dark ports. As illustrated in Fig. 2, the saddle points of the particle spectrum represent a choice of the set of parameters $\left(\kappa, \tau_{s}, l\right)$ at which the visibility of the particle spectrum is maximized.

\section{CONCLUDING REMARKS}

We have suggested at the theoretical level a potential application of quantum interferometry in the realm of macroscopic quantum mechanics, with a view that these experiments may be considered in conjunction with the prototype testing in the development of an advanced gravitational wave detector. We have not considered feasible experimentally accessible parameters of the proposed experiment, as it seems to us that at this preliminary stage of investigation it is premature to do so. To carry out the experiment we have to prepare the mirror in its ground state in the first place. Recent experimental efforts [19-21] suggest that cooling the mirror down to its ground state is feasible and would not become an insurmountable obstacle. However it is likely to take some time to accomplish this goal. Further, like other related proposed experiments in testing macroscopic quantum mechanics [4,9], among all conceivable experimental problems, obtaining the desired coherence time for a single photon and preventing the generation of spurious phonons due to excitation of internal degrees of freedom of the mirror are the two most challenging tasks as far as we see. Though it is unlikely that the suggested experiment is to be realised in the near future, it represents a lofty goal toward which we expend our effort in the foreseeable future.

\section{ACKNOWLEDGMENTS}

Y.K.L. is grateful to Yanbei Chen for useful discussions and inspiration. The work is supported by NBRP of China 2010CB832800, NNSF of China 10505009, NSFC 10731080, and the 973 project under Contract No. 2006CB805905.

\section{APPENDIX: MICHELSON INTERFEROMETRY}

Upon the completion of our study of Saganc interferometry, it is a natural question to ask whether the test of commutation relation of a macroscopic quantum mirror can only be done solely by the Sagnac setup. To understand this question better, we also look into the familiar Michelson setup. It turns out that the test of commutation relation is not unique to the Sagnac setup. A similar conclusion may also be drawn for the Michelson setup and is already implicit in [4]. We shall briefly sketch the probe of commutation relation for a macroscopic mirror using Michelson interferometry in this appendix.

Consider the standard Michelson setup as shown in Fig. 3 [4] with only one single photon entering the interferometer. The end mirror in the north arm is assumed to be movable while the mirror in the east arm is rigidly attached.

Let $a_{1}, a_{0}$ be the annihilation operators of single photon input field and vacuum input field (see Fig. 3) and $a_{N}, a_{E}$ the annihilation operators of the field modes going into north and east arms. A balanced beamsplitter then yields

$$
\left(\begin{array}{l}
a_{N} \\
a_{E}
\end{array}\right)=\frac{1}{\sqrt{2}}\left(\begin{array}{cc}
1 & 1 \\
1 & -1
\end{array}\right)\left(\begin{array}{l}
a_{1} \\
a_{0}
\end{array}\right)
$$

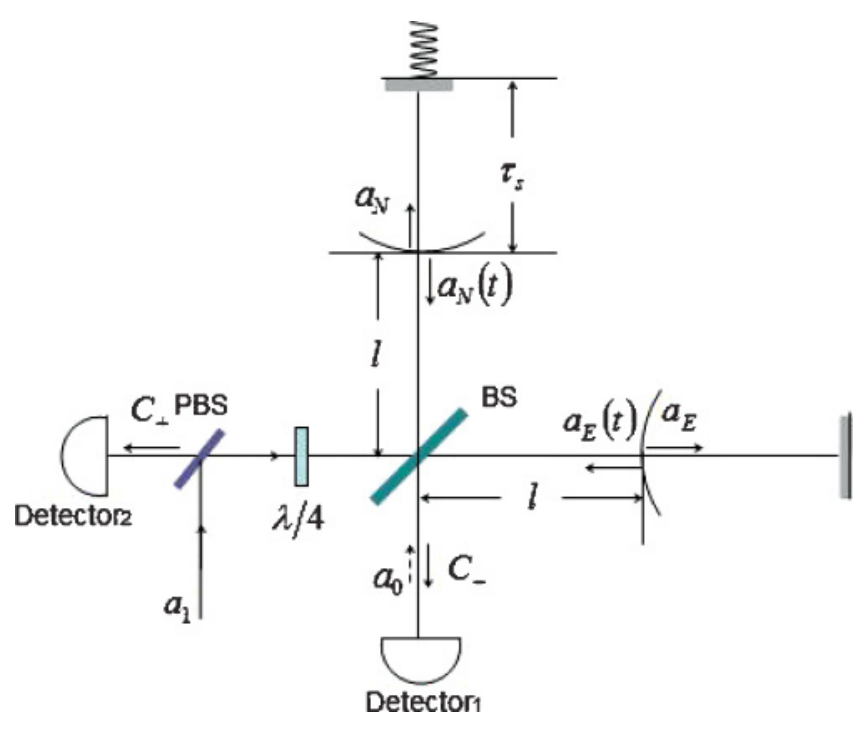

FIG. 3. (Color online) Michelson interferometry. 
The Hamiltonian of the total system including optomechanical coupling may be given as

$$
\begin{aligned}
H= & \omega a_{N}^{\dagger} a_{N}+\omega a_{E}^{\dagger} a_{E}+\omega_{m} b^{\dagger} b \\
& -g a_{N}^{\dagger} a_{N}\left(b^{\dagger}+b\right) \theta\left(l, l+\tau_{s}\right) .
\end{aligned}
$$

The above Hamiltonian was considered in [4] (except with the step function made implicit), in which the reduced single photon density matrix at the readout was worked out. Our contribution in this appendix lies in working out the inputoutput relation of the interferometer in terms of the Heisenberg picture. We may see clearly that apart from the linear superposition of a macroscopic mirror, the particle spectrum also encodes information concerning the commutation relation of the freely movable mirror. The role of the commutation relation in the readout is quite evident when looking at the problem from the Heisenberg picture. The same piece of information seems to be difficult to extract from the Schrödinger picture.

Subject to the Hamiltonian in (A1), for the time $t=\tau>$ $2 l+\tau_{s}$, the Heisenberg equations of motion yield

$$
\begin{aligned}
& a_{N}(\tau)=a_{N}(0) e^{-i \omega \tau} e^{i 2 \kappa^{2} a_{N}^{\dagger} a_{N}\left(\omega_{m} \tau_{s}-\sin \omega_{m} \tau_{s}\right)} \\
& \times e^{\kappa b(0)^{\dagger} e^{i \omega m l}\left(e^{i \omega m \tau_{s}}-1\right)-\kappa b(0) e^{-i \omega m l}\left(e^{-i \omega m \tau_{s}}-1\right)}, \\
& a_{E}(\tau)=a_{E}(0) e^{-i \omega \tau}, \\
& b(\tau)=b(0) e^{-i \omega_{m} \tau}+\kappa a_{N}^{\dagger} a_{N}\left(1-e^{-i \omega_{m} \tau_{s}}\right) .
\end{aligned}
$$

As may be checked from (A1) and (A2), the number operators $a_{N}^{\dagger} a_{N}$ and $a_{E}^{\dagger} a_{E}$ are QND observables.

Similar to the Sagnac case, at the bright (+) and dark (-) ports, we have

$$
\left(\begin{array}{l}
c_{+}(\tau) \\
c_{-}(\tau)
\end{array}\right)=\frac{1}{\sqrt{2}}\left(\begin{array}{cc}
1 & 1 \\
1 & -1
\end{array}\right)\left(\begin{array}{l}
a_{N}(\tau) \\
a_{E}(\tau)
\end{array}\right),
$$

and the corresponding number operators $N_{ \pm}$defined in (9).
Consider the initial state of the coupled photon-mirror system to be $\frac{1}{\sqrt{2}}\left(|1\rangle_{N}|0\rangle_{E}+|0\rangle_{N}|1\rangle_{E}\right)|0\rangle_{m}$ with $|0\rangle_{m}$ being the ground state of the mirrors. Given (A2), like in the Sagnac case, the expectation values of the two number operators may be worked out to be

$$
\left\langle N_{ \pm}(t)\right\rangle=\frac{1}{2} \pm \frac{1}{2} e^{-\kappa^{2}\left(1-\cos \omega_{m} \tau_{s}\right)} \cos \left[\kappa^{2}\left(\omega_{m} \tau_{s}-\sin \omega_{m} \tau_{s}\right)\right] .
$$

In the calculations leading up to (A4), we note that the term $e^{-\kappa^{2}\left(1-\cos \omega_{m} \tau_{s}\right)}$ comes from

$$
\begin{aligned}
e^{\kappa} & b(0)^{\dagger} e^{i \omega_{m} l}\left(e^{i \omega_{m} \tau_{s}}-1\right)-\kappa b(0) e^{-i \omega_{m} l}\left(e^{-i \omega_{m} \tau_{s}}-1\right) \\
& =e^{i \kappa^{2}\left(1-\cos \omega_{m} \tau_{s}\right)\left[x_{0}, p_{0}\right]} e^{\kappa b(0)^{\dagger} e^{i \omega_{m} l}\left(e^{i \omega_{m} \tau_{s}}-1\right)} e^{-\kappa b(0) e^{-i \omega_{m} l}\left(e^{-i \omega_{m} \tau_{s}}-1\right)} \\
& =e^{-\kappa^{2}\left(1-\cos \omega_{m} \tau_{s}\right)} e^{\kappa b(0)^{\dagger} e^{i \omega_{m} l}\left(e^{i \omega_{m} \tau_{s}}-1\right)} e^{-\kappa b(0) e^{-i \omega_{m} l}\left(e^{-i \omega_{m} \tau_{s}}-1\right)},
\end{aligned}
$$

where the second equality follows from the commutation relation $\left[x_{0}, p_{0}\right]=i$. So again the particle spectrum encodes information of the commutation relation of a macroscopic mirror. However, while the entire particle spectrum in the Sagnac case originates from the commutation relation, a slight difference in the Michelson case is that the modulation term $\cos \left[\kappa^{2}\left(\omega_{m} \tau_{s}-\sin \omega_{m} \tau_{s}\right)\right]$ is not related to the commutation relation of the macroscopic mirror but is instead generated from the expectation values of other operator terms in $N_{ \pm}$.

Moreover, the nonconstant term in the right-hand side of (A4) may be identified with the visibility factor in [4]. In this sense the noncommutativity is already implicit in the previous work. Our calculations serve to bring out in an explicit manner the dependence of the readout particle spectrum on the commutation relation of a macroscopic mirror. As a result, we see that, apart from being a way to read the linear superposition of a macroscopic quantum mirror, single photon Michelson interferometry at the same time also provides a direct probe to the commutation relation of a macroscopic mirror.
[1] M. Arndt, O. Nairz, J. Vos-Andreae, C. Keller, G. van der Zouw, and Anton Zeilinger, Nature (London) 401, 680 (1999).

[2] C. H. van der Wal, A. C. J. ter Haar, F. K. Wilhelm, R. N. Schouten, C. J. P. M. Harmans, T. P. Orlando, S. Lloyd, and J. E. Mooij, Science 290, 773 (2000).

[3] R. Penrose, The Road to Reality (Vintage Press, London, 2005).

[4] W. Marshall, C. Simon, R. Penrose, and D. Bouwmeester, Phys. Rev. Lett. 91, 130401 (2003).

[5] S. Mancini, V. Giovannetti, D. Vitali, and P. Tombesi, Phys. Rev. Lett. 88, 120401 (2002).

[6] H. Müller-Ebhardt, H. Rehbein, R. Schnabel, K. Danzmann, and Y. Chen, Phys. Rev. Lett. 100, 013601 (2008).

[7] O. Arcizet, P.-F. Cohadon, T. Briant, M. Pinard, and A. Heidmann, Nature (London) 444, 71 (2006).

[8] C. K. Law, Phys. Rev. A 49, 433 (1994).

[9] S. Bose, K. Jacobs, and P. L. Knight, Phys. Rev. A 56, 4175 (1997).

[10] V. Parigi, A. Zavatta, M. S. Kim, and M. Bellini, Science 317, 1890 (2007).

[11] M. S. Kim, H. Jeong, A. Zavatta, V. Parigi, and M. Bellini, Phys. Rev. Lett. 101, 260401 (2008).
[12] Y. Chen, Phys. Rev. D 67, 122004 (2003).

[13] V. B. Braginsky and F. Ya. Khalili, Quantum Measurement (Cambridge University Press, Cambridge, 1992).

[14] S. Hild, S. Chelkowski, and A. Freise, e-print arXiv:0810.0604v2 [gr-qc].

[15] A. Freise, S. Chelkowski, S. Hild, W. Del Pozzo, A. Perreca, and A. Vecchio, Class. Quantum Grav. 26, 085012 (2009).

[16] R. W. P. Drever, in Gravitational Radiation, edited by N. Deruelle and T. Piran (North-Holland, Amsterdam, 1983), pp. 321-338.

[17] S. L. Danilishin, Phys. Rev. D 69, 102003 (2004).

[18] Ziren Luo, Ran Yang, Xuefei Gong, Shouyong Pei, and Y. K. Lau (in preparation).

[19] S. Gröblacher et al., Nature Phys. 5, 485 (2009).

[20] D. E. Chang, C. A. Regal, S. B. Papp, D. J. Wilson, J. Ye, O. Painter, H. J. Kimble, and P. Zoller, e-print arXiv:0909.1548v2.

[21] O. Romero Isart, M. L. Juan, R. Quidant, and J. Ignacio Cirac, New J. Phys. 12, 033015 (2010). 